\title{
Steering nonholonomic systems in chained form*
}

\author{
Richard M. Murray ${ }^{\dagger} \quad$ S. Shankar Sastry \\ Department of Electrical Engineering and Computer Sciences \\ University of California Berkeley, CA 94720
}

\begin{abstract}
In this paper we introduce a nilpotent form, called chained form, for nonholonomic control systems. For the case of a nonholonomic system with two inputs, we give constructive conditions for the existence of a feedback transformation which puts the system into chained form, and show how to steer the system between arbitrary states. Examples are presented for steering a car and a car with a trailer attached; other examples can be found in the areas of space robotics and multi-fingered robot hands. The results of this paper also have applications in the area of nilpotentization of distributions of vector fields on $\mathbf{R}^{n}$.
\end{abstract}

\section{Introduction}

Consider the problem of steering a system with configuration $x \in$ $\mathbb{R}^{n}$ subject to a set of independent kinematic constraints having the form

$$
\omega_{j}(x) \dot{x}=0 \quad j=1, \cdots, k .
$$

We assume the $\omega_{j}$ 's are smooth and linearly independent over the ring of smooth functions. Formally, these constraints are exterior differential one-forms on $\mathbb{R}^{n}$. Such constraints can arise when two surfaces roll against each other, such as the rolling between a wheel and the road, or in space-based systems where the total angular momentum of the system is conserved. Although strictly speaking this latter case is not a "constraint", it can be treated with the same set of tools.

To study such a system, we convert the path planning problem into a control problem. Let $\Delta$ be a distribution of dimension $m=n-k$ which is annihilated by the constraints. We represent this distribution with respect to a basis of vector fields:

$$
\Delta=\operatorname{span}\left\{g_{1}, g_{2}, \cdots, g_{m}\right\} \quad g_{i}(x) \in \mathbb{R}^{n}
$$

In coordinates, the constraint one-forms can be written as an $k \times n$ matrix and the $g_{i}$ 's are a basis for the right null space of this matrix. The path planning problem can then be restated as finding an input function, $u(t) \in \mathbb{R}^{m}$ such that the control system

$$
\dot{x}=g_{1}(x) u_{1}+\cdots+g_{m}(x) u_{m}
$$

is driven from $x_{0}$ to $x_{\mathbf{1}}$. As a consequence of our assumptions on the $\omega_{j}$ 's, the $g_{i}$ 's are also smooth and linearly independent.

The condition for the existence of a path between two configurations is given by Chow's theorem. We let $[f, g]$ be the Lie bracket between two vector fields,

$$
[f, g]=\frac{\partial g}{\partial x} f-\frac{\partial f}{\partial x} g,
$$

${ }^{*}$ Research supported in part by the National Science Foundation under grant IRI-90-14490

tCurrently with the Department of Mechanical Engineering, California Institute of Technology, Pasadena, CA 91125

$\mathrm{CH} 3076-7 / 91 / 0000-\$ 121 \$ 01.00$ (C) 1991 IEEE and define the involutive closure of a distribution $\Delta$ as the closure of $\Delta$ under Lie bracketing. Briefly, Chow's theorem states that if the involutive closure of the distribution associated with equation (3) spans $\mathbb{R}^{n}$ at each configuration, the system can be steered between any two configurations. It is not apparent how the path can be explicitly constructed; in this paper we propose techniques for generating such paths.

We say that a system is holonomic if the kinematic constraints in (1) restrict the motion of the system to a manifold of dimension $n-k$. In this case, the constraints on the system can be rewritten as an algebraic constraint on the configuration variables $x$. A system is nonholonomic if it is not constrained to lie on a manifold of the same dimension as the input space. In particular, we are most interested in systems which are maximally nonholonomic: any point in the configuration space can be reached. This is equivalent to saying that the corresponding control system is controllable. If the system is not maximally nonholonomic, it can still be treated by restricting the initial and final configurations to lie on the same leaf of the foliation generated by the distribution.

It is possible to classify nonholonomic systems based on the way in which controllability is achieved. Define $G_{1}=\Delta$ and

$$
G_{i}=G_{i-1}+\left[G_{1}, G_{i-1}\right]
$$

where

$$
\left[G_{1}, G_{i-1}\right]=\operatorname{span}\left\{[g, h]: g \in G_{1}, h \in G_{i-1}\right\}
$$

A system is regular in a neighborhood $U$ of $x_{0}$ if

$$
\operatorname{rank} G_{i}(x)=\operatorname{rank} G_{i}\left(x_{0}\right) \quad \forall x \in U .
$$

If a system is regular, there exists an integer $p<n$ such that $G_{i}=G_{p+1}$ for all $i \geq p+1$. We refer to $p$ as the degree of nonholonomy of the distribution. The growth vector for a regular system is defined as $r \in \mathbb{Z}^{p+1}$, where $r_{i}=$ rank $G_{i}$. We define the relative growth vector $\sigma \in \mathbb{Z}^{p+1}$ as $\sigma_{i}=r_{i}-r_{i-1}$ and $r_{0}:=0$. The growth vector for a (regular) system is a convenient way to represent information about the associated control Lie algebra. See $[5,21]$ for more details.

Nonholonomic control systems cannot be studied using the usual linear control techniques. The linearization of a nonholonomic control system is degenerate; linearizing the system about a point gives:

$$
\dot{x}=A x+B u \quad A=0, B=\left[g_{1}(0) \cdots g_{m}(0)\right]
$$

which is clearly not controllable. Furthermore, the feedback linearization conditions fail for such systems due to the lack of a term which is independent of $u$. In particular, if the system is controllable, the distribution

$$
\Delta_{0}=\operatorname{span}\left\{g_{1}, g_{2}\right\}
$$

is not involutive, violating the necessary conditions for full state feedback linearization [8]. 


\section{Related work}

There has been a large interest in the control of nonholonomic systems in the control and robotics literature. We mention here only a few of the papers which have influenced our work. A more complete set of citations can be found in $[19,22]$.

Much of the early work in nonholonomic motion planning was devoted to path planning for mobile robots. Laumond used a set of canonical paths to steer a cart, and later a cart with a trailer, to an arbitrary location in the presence of obstacles $[13,14,15$. More recent work has been used for a mobile robot with bounded input constraints in addition to the nonholonomic constraint [9]. The approach presented in this latter paper may be applicable to more general systems. Another general algorithm, developed by Barraquand and Latombe, can reportedly handle any set of nonholonomic constraints [1]. Sample paths are presented for a frontwheel drive car and a car pulling a trailer. The paths generated are locally time-optimal, but can be computed only in sufficiently low-dimensional spaces. Yet another approach has been to train a neural net to park a car pulling a trailer [23].

Control theoretic approaches to to nonholonomic motion planning problems have also been explored, beginning with the work of Brockett [4]. Brockett showed that for a class of systerns with degree of nonholonomy 1 , the optimal controls consist of sinusoids at integrally related frequencies. In the robotics lit erature, Li and Canny studied the motion of a fingertip rolling on an object without slipping [16]. This problem has also been investigated using some of the methods presented here [20]. Later work by $\mathrm{Li}$ and others studied a hopping robot flipping in mid-air by using conservation of angular momentum to construct paths on a reduced space [17]. Similar techniques have also been used for studying the motion of coupled rigid bodies and space manipulators $[25,18]$. Sussmann et al. have recently used Lie algebraic techniques for generating nonholonomic motions [12] and for approximating arbitrary trajectories with feasible ones [26].

\section{Overview}

The paper is organized as follows. Section 2 reviews our previous results in steering nonholonomic systems and constructs a special class of nonholonomic systems, called chained systems. Using the concept of chained systems, we give an algorithm which can be used to steer a system in chained form to an arbitrary location. Section 3 considers the problem of converting a nonholonomic system into chained form. We consider only the 2 input case, since most of the examples which have motivated our research are of this form. Section 3 also contains examples of how to convert systems into chained form.

\section{Chained form}

In our previous work [21], we used sinusoidal inputs to steer a class of nonholonomic systems. These systems had a special triangular form which allowed Fourier series techniques to be used to analyze the motion resulting from inputs which consisted of sines and cosines at integrally related frequencies. As a simple example, consider the system

$$
\begin{aligned}
& \dot{x}_{1}=u_{1} \\
& \dot{x}_{2}=u_{2} \\
& \dot{x}_{3}=x_{2} u_{1} \\
& \dot{x}_{4}=x_{3} u_{1} \\
& \dot{x}_{5}=x_{3} u_{1}
\end{aligned}
$$

For this system, the distribution

$$
\left\{g_{1}, g_{2},\left[g_{1}, g_{2}\right],\left[g_{1},\left[g_{1}, g_{2}\right]\right],\left[g_{2},\left[g_{1}, g_{2}\right]\right]\right\}
$$

spans $\mathbb{R}^{5}$ and hence the system is controllable. The system has degree of nonholonomy 2 since it takes two levels of brackets to span the tangent space to the configuration manifold.

This system can be steered using sinusoidal inputs. The algorithm proceeds as follows. Using constant inputs, steer $x_{1}$ and $x_{2}$ to their desired values. During this motion, $x_{3}, x_{4}$ and $x_{5}$ will drift. Next, use $u_{1}=a_{3} \sin t$ and $u_{2}=b_{3} \cos t$ to move $x_{3}$ to its desired value. These inputs describe a closed loop in $x_{1}$ and $x_{2}$ and hence at the end of this motion the first 3 variables have obtained their desired values. Finally, using $u_{1}=a_{4} \sin t, u_{2}=b_{4} \cos 2 t$ and $u_{1}=a_{5} \cos 2 t, u_{2}=b_{5} \sin t$, it is possible to steer $x_{4}$ and $x_{5}$ while leaving all other states unchanged. Hence we have suc ceeded in steering the system to its desired location. Full details are contained in [21].

There are many advantages to using sinusoidal inputs for steering nonholonomic systems. The paths generated are piece wise smooth and consist of a relatjvely small number of segments ( 4 for the example above). Furthermore, the use of sinusoids allows some nonlinear systems to be treated with minor modifications to the algorithm given above. This leads to exact steering algorithms for certain nonlinear systems, in contrast to the approximate (iterative) method proposed by Lafferriere and Sussmann (for nonnilpotent systems).

It can be shown that simple sinusoids cannot be effectively used for steering arbitrary nonholonomic systems. In particular, it is possible to build systems using techniques formulated by Grayson and Grossman [6] which cannot be steered using sinusoidal inputs at single frequencies $[19,22]$. The construction of these systems relies on the use of a $P$. Hall basis for a Lie algebra of with a fixed number of generators and a given degree of nilpotency.

Rather than explore the use of more complicated inputs for steering nonholonomic systems, we consider instead a simpler class of systems. The justification for changing the class of systems is simple-most of the systems encountered as examples do not have the complicated structure of the general case. Thus there may be a simpler class of systems which is both steerable using simple sinusoids and representative of systems in which we are interested.

Consider a two input system of the following form:

$$
\begin{array}{rlrl}
\dot{x}_{0} & =u_{1} & \dot{y}_{0} & =u_{2} \\
\dot{x}_{1} & =y_{0} u_{1} & \left(y_{1}:=-x_{1}\right) \\
\dot{x}_{2} & =x_{1} u_{1} & \dot{y}_{2} & =y_{1} u_{2} \\
\dot{x}_{3} & =x_{2} u_{1} & \dot{y}_{3} & =y_{2} u_{2} \\
\vdots & \vdots & \vdots \\
\dot{x}_{n_{x}} & =x_{n_{x-1}} u_{1} & \dot{y}_{n_{y}} & =y_{n_{y}-1} u_{2}
\end{array}
$$

or more compactly

$$
\begin{aligned}
\left(\begin{array}{c}
\dot{x} \\
\dot{y}
\end{array}\right)=X u_{1}+Y u_{2} \quad & =\frac{\partial}{\partial x_{0}}+y_{0} \frac{\partial}{\partial x_{1}}+\sum_{i=2}^{n} x_{i-1} \frac{\partial}{\partial x_{i}} \\
Y & =\frac{\partial}{\partial y_{0}}+\sum_{j=2}^{n} y_{j-1} \frac{\partial}{\partial y_{j}}
\end{aligned}
$$

where $y_{1}:=-x_{1}$ to account for skew-symmetry of the Lie bracket. We refer to this system as a two-chain system. The first item is to check the controllability of these systems. To this end, denote iterated Lie products as $\operatorname{ad}_{X}^{k} Y$ :

$\operatorname{ad}_{X} Y=[X, Y] \quad \operatorname{ad}_{X}^{k} Y=\left[X, \operatorname{ad}_{X}^{k-1} Y\right]=[X,[X, \cdots,[X, Y] \cdots]]$ 
Lemma 1 (Lie bracket calculations)

For the vector fields in equation (4)

$$
\begin{aligned}
\operatorname{ad}_{X}^{k} Y & =(-1)^{k} \frac{\partial}{\partial x_{k}} \quad k>1 \\
\operatorname{ad}_{Y}^{k} X & =(-1)^{k} \frac{\partial}{\partial y_{k}}
\end{aligned}
$$

Proof. By induction. Since the first level of brackets is irregular, we begin by expanding $[X, Y]$ and $[X,[X, Y]]$.

$$
\begin{aligned}
{[X, Y]=} & \left(\frac{\partial}{\partial x_{0}}+y_{0} \frac{\partial}{\partial x_{1}}+\sum x_{i-1} \frac{\partial}{\partial x_{i}}\right)\left(\frac{\partial}{\partial y_{0}}+\sum y_{j-1} \frac{\partial}{\partial y_{j}}\right) . \\
& \left(\frac{\partial}{\partial y_{0}}+\sum y_{j-1} \frac{\partial}{\partial y_{j}}\right)\left(\frac{\partial}{\partial x_{0}}+y_{0} \frac{\partial}{\partial x_{1}}+\sum x_{i-1} \frac{\partial}{\partial x_{i}}\right) \\
=0 & -\frac{\partial}{\partial x_{1}} \\
{[X,[X, Y]]=} & X\left(-\frac{\partial}{\partial x_{1}}\right)+\frac{\partial}{\partial x_{1}}(X)=0+\frac{\partial}{\partial x_{2}}
\end{aligned}
$$

Now assume that $\operatorname{ad}_{X}^{k} Y=(-1)^{k} \frac{\partial}{\partial x_{k}}$. Then

$$
\begin{aligned}
\operatorname{ad}_{X}^{k+1} Y & =\left[X, \operatorname{ad}_{X}^{k} Y\right] \\
& =(-1)^{k}\left(X\left(\frac{\partial}{\partial x_{k}}\right)-\frac{\partial}{\partial x_{k}}(X)\right)=(-1)^{k+1} \frac{\partial}{\partial x_{k+1}}
\end{aligned}
$$

The proof for $\operatorname{ad}_{Y}^{k} X$ is identical using the facts $[Y, X]=-[X, Y]$ and $y_{1}:=\cdots x_{1}$.

\section{Proposition 1 (Controllability of the two-chain system)} The two-chain system (4) is maximally nonholonomic (controllable).

Proof. There are $2 n-1$ coordinates in (4) and the $2 n-1$ Lie products

$$
\left\{X, Y, \operatorname{ad}_{X}^{i} Y, \operatorname{ad}_{Y}^{j} X\right\} \quad i \geq 1, \quad j \geq 2
$$

are independent using Lemma 1 . We require $j \geq 2 \operatorname{since} \operatorname{ad}_{Y} X=$ $-\operatorname{ad}_{X} Y$ and hence those Lie products can never be independent.

To steer this system, we use sinusoids at integrally related frequencies. Roughly speaking, if we use $u_{1}=\sin t$ and $u_{2}=\cos k t$ then $\dot{x}_{1}$ will have components at frequency $k-1, \dot{x}_{2}$ at frequency $k-2$, etc. $\dot{x}_{k}$ will have a component at frequency zero and when integrated we get motion in $x_{k}$ while all previous variables return to their starting values. In the $y$ variables, all frequency compo nents will be of the form $m \cdot k \pm 1$ and hence we get no motion for $k>1$. (For $k=1, y_{1}$ and $x_{1}$ are the same variable). We make this precise with the following algorithm

\section{Algorithm 1}

1. Steer $x_{0}$ and $y_{0}$ to their desired values.

2. For each $x_{k}, k \geq 1$, steer $x_{k}$ to its final value using $u_{1}=a \sin t$, $u_{2}=b \cos k t$, where $a$ and $b$ satisfy

$$
x_{k}(2 \pi)-x_{k}(0)=\frac{(a / 2)^{k} b}{k !} \cdot 2 \pi
$$

3. For each $y_{k}, k \geq 2$, steer $y_{k}$ to its final value using $u_{1}=$ $b \cos k t, u_{2}=a \sin t$, where $a$ and $b$ satisfy

$$
y_{k}(2 \pi)-y_{k}(0)=\frac{(a / 2)^{k} b}{k !} \cdot 2 \pi
$$

Proposition 2 Algorithm 1 can steer (4) to an arbitrary configuration.

Proof. The proof is constructive. It suffices to consider only step 2 since step 3 can be proved by switching $x$ and $y$ in what follows. We must show 2 things:

1. moving $x_{k}$ does not affect $x_{j}, j<k$

2. moving $x_{k}$ does not affect $y_{j}, j=1, \cdots, n_{y}$

To verify that using $u_{1}=a \sin t, u_{2}=b \cos k t$ produces motion only in $x_{k}$, we integrate the $x$ states. If $x_{k-1}$ has terms at frequency $\omega_{i}$, then $x_{k}$ has corresponding terms at $\omega_{i} \pm 1$ (by expanding products of sinusoids as sums of sinusoids). Since the only way to have $x_{i}(2 \pi) \neq x_{i}(0)$ is to have $x_{i}$ have a component at frequency zero, it suffices to keep track only of the lowest frequency component in each variable; higher components will integrate to zero. Direct computation starting from the origin yields

$$
\begin{aligned}
x_{0} & =a(1-\cos t) \\
x_{1} & =\int \frac{a b}{k} \sin k t \sin t \\
& =\frac{1}{2} \frac{a b}{k(k-1)} \sin (k-1) t+\frac{1}{2} \frac{a b}{k(k+1)} \sin (k+1) t \\
x_{2} & =\frac{1}{2^{k}} \frac{a^{2} b}{k(k-1)(k-2)} \sin (k-2) t+\cdots \\
& \vdots \\
x_{k} & =\int\left(\frac{a^{k} b}{2^{k-1} k !} \sin ^{2} t+\cdots\right) d t=\frac{a^{k} b}{2^{k-1} k !} \frac{t}{2}+\cdots
\end{aligned}
$$

$x_{k}(2 \pi)=x_{k}(0)+\frac{(a / 2)^{k} b}{k k^{\prime}} \pi$ and all earlier $x_{i}$ 's are periodic and hence $x_{i}(2 \pi)=x_{i}(0), i<k$. If the system does not start at the origin, the initial conditions generate extra terms of the form $x_{i-1}(0) u_{2}$ in the $i^{\text {th }}$ derivative and this integrates to zero, giving no net contribution.

To show that we get no motion in the $y$ variables, we show that all frequency components in the $y$ 's have the form $m k \pm 1$ where $m$ is some integer. This is true for $y_{1}:=-x_{1}$ from the calculation above. Assume it is true for $y_{i}$ :

$$
\begin{aligned}
\dot{y}_{i+1} & =y_{i} u_{2} \\
& =\sum_{m} \alpha(m) \sin (m k \pm 1) t \cdot \cos k t \\
& =\sum_{m} \frac{\alpha(m)}{2}(\sin ((m+1) k \pm 1) t+\sin ((m-1) k \pm 1) t)
\end{aligned}
$$

Hence $y_{i+1}$ only has components at non-zero frequencies $m^{\prime} k \pm 1$ and therefore $y_{i}(2 \pi)=y_{1}(0)$.

To include systems with more than two inputs, we replicate the structure of (4) for each additional input. Let $h_{i j}^{k}$ represent the motion corresponding to the Lie product $\operatorname{ad}_{x}^{k} X_{j}$. In the two input case, $x_{k}=h_{21}^{k}$ and $y_{k}=h_{12}^{k}$. The following system on $\mathbb{R}^{n}$ is the m-chain system:

$$
\begin{array}{rlrl}
\dot{h}_{j}^{0} & =u_{j} & j=1, \cdots, m \\
\dot{h}_{i j}^{1} & =h_{i}^{0} u_{j} & & i>j \text { and } h_{j i}^{1}:=-h_{i j}^{1} \\
\dot{h}_{i j}^{k} & =h_{i j}^{k-1} u_{j} & &
\end{array}
$$

Proposition 3 (Multi-chain system controllability) The multi-chain system of (5) is maximally nonholonomic and can be steered using sinusoids. 
Proof. The system (5) can be rewritten

$$
\dot{h}=X_{1} u_{1}+\cdots+X_{m} u_{m}
$$

with

$$
X_{j}=\frac{\partial}{\partial h_{j}^{0}}+\sum_{\substack{i=1 \\ i<j}}^{m} h_{i}^{0} \frac{\partial}{\partial h_{i j}^{1}}+\sum_{k} \sum_{i} h_{i j}^{k-1} \frac{\partial}{\partial h_{i j}^{k}}
$$

Given any two $X_{i}, X_{j}$, their Lie product expansions only involve terms of the form $h_{i j}^{k}$ for some $k$. But this is precisely the vector fields from Lemma 1 and hence

$$
\operatorname{ad}_{X_{i}}^{k} X_{j}=(-1)^{k} \frac{\partial}{\partial h_{i j}^{k}}
$$

Taking these terms for all possible $i, j, k$ we get a set of independent Lie products just as in the proof of Theorem 1.

To show that the system can be steered using sinusoids, pick any $i, j \in\{1, \cdots, m\}, i<j$. Fix $u_{i}=0$ for all $l \neq i, j$. The resulting system is identical to (4) can be steered using algorithm 1. By choosing all possible combinations of $i$ and $j$, we can move to any position.

\section{Converting Systems to Chained Form}

In this section we introduce a set of sufficient conditions for determining if a system can be converted to chained form. This set of conditions gives a constructive method for building a feedback transformation which accomplishes the conversion. We concentrate on the two input case with a single chain.

Proposition 4 (Converting systems to two-chained form) Consider a controllable system

$$
\dot{x}=g_{1}(x) u_{1}+g_{2}(x) u_{2}
$$

with $g_{1}, g_{2}$ linearly independent and smooth and having the special form

$$
\begin{aligned}
& g_{1}(x)=\frac{\partial}{\partial x_{1}}+\sum_{i=2}^{n} g_{1}^{i}(x) \frac{\partial}{\partial x_{i}} \\
& g_{2}(x)=\sum_{i=2}^{n} g_{2}^{i}(x) \frac{\partial}{\partial x_{i}}
\end{aligned}
$$

(by appropriate change of basis, if necessary). Define

$$
\begin{aligned}
& \Delta_{0}:=\operatorname{span}\left\{g_{1}, g_{2}, \operatorname{ad}_{g_{1}} g_{2}, \cdots, \operatorname{ad}_{g_{1}}^{n-2} g_{2}\right\} \\
& \Delta_{1}:=\operatorname{span}\left\{g_{2}, \operatorname{ad}_{g_{1}} g_{2}, \cdots, \operatorname{ad}_{g_{1}}^{n-3} g_{2}\right\}
\end{aligned} .
$$

If for some open set $U, \Delta_{0}(x)=\mathbf{R}^{n}$ for all $x \in U \subset \mathbb{R}^{n}$ and $\Delta_{1}$ is involutive on $U$, then there exists a local feedback transformation

$$
\xi=\phi(x) \quad u=\beta(x) v
$$

such that the transformed system is in chained form:

$$
\begin{aligned}
& \dot{\xi}_{1}=v_{1} \\
& \dot{\xi}_{2}=v_{2} \\
& \dot{\xi}_{3}=\xi_{2} v_{1} \\
& \quad \vdots \\
& \dot{\xi}_{n}=\xi_{n-1} v_{1}
\end{aligned}
$$

Proof. Since $\Delta_{1}$ is an involutive distribution of dimension $n-2$, there exists a function $h$ such that $d h \cdot \Delta_{2}=0$ and $d h \cdot \operatorname{ad}_{g_{1}}^{n-2} g_{2} \neq 0$. Define the map $\phi: x \mapsto \xi$ as

$$
\begin{aligned}
\xi_{1} & =x_{1} \\
\xi_{2} & =L_{g_{1}}^{n-2} h \\
\vdots & \\
\xi_{n-1} & =L_{g_{1}} h \\
\xi_{n} & =h
\end{aligned}
$$

To verify that $\phi$ is a valid change of coordinates, we use the fact that

so that

$$
L_{[f, g]} h=L_{f} L_{g} h-L_{g} L_{f} h
$$

$$
\begin{aligned}
L_{a d_{g_{1}}^{n-2} g_{2}} h & =L_{g_{1}} L_{a d_{g_{1}}^{n-3} g_{2}} h-L_{a d_{g_{1}}^{n-3} g_{2}} L_{g_{1}} h \\
& =(-1)^{n-2} L_{g_{2}} L_{g_{1}}^{n-2} h \neq 0
\end{aligned}
$$

and $L_{a d_{1}^{k} g_{2}} h=0$ for $k<n-2$ by the same reasoning. Using this calculation,

$\frac{\partial \phi}{\partial x}=\left[\begin{array}{c}d h \\ d L_{g_{1}}^{n-2} h \\ \vdots \\ d L_{g_{1}} h\end{array}\right] \quad \frac{\partial \phi}{\partial x} \Delta_{0}=\left[\begin{array}{ccccc}1 & 0 & 0 & \ldots & 0 \\ * & \pm a(x) & * & \ldots & * \\ * & 0 & \pm a(x) & & \vdots \\ \vdots & \vdots & & \ddots & * \\ * & 0 & \ldots & 0 & \pm a(x)\end{array}\right]$

where $a(x)=L_{g_{2}} L_{g_{1}}^{n-2} h \neq 0$.

Evaluating the derivatives of the coordinate transformation, we define

$$
\begin{aligned}
& v_{1}:=u_{1} \\
& v_{2}:=\left(L_{g_{1}}^{n-1} h\right) u_{1}+\left(L_{g_{2}} L_{g_{1}}^{n-2} h\right) u_{2}
\end{aligned} .
$$

Since $L_{g_{2}} L_{g_{1}}^{n-2} h \neq 0$, this change of inputs is invertible and the resulting system is in chained form.

This proposition gives a set of sufficient conditions for converting a system with relative growth vector $\sigma=(2,1, \cdots, 1)$ into chained form (locally). In order to apply the results, however, we must modify the original inputs to the system such that one of the states is controlled directly by the input. Such a change of input is always possible due to the assumption that the input vector fields are linearly independent. This change of input is not unique.

One corollary to Proposition 4 is that all systems with relative growth vector $\sigma=(2,1)$ can be converted to chained form. This is a direct consequence of the fact that all 1 dimensional distributions are involutive.

Example 1 (Kinematic car) Consider as our first example, the kinematic model of an automobile. The equations governing the motion of the system are [22]:

$$
\begin{aligned}
\dot{x} & =\cos \theta u_{1} \\
\dot{y} & =\sin \theta u_{1} \\
\dot{\phi} & =u_{2} \\
\dot{\theta} & =\frac{1}{l} \tan \phi u_{1}
\end{aligned}
$$

To convert the system to chained form, we first scale the inputs so that $u_{1}$ enters $\dot{x}$ directly. Reusing the symbol $u_{1}$, the kinematics become:

$$
\begin{aligned}
\dot{x} & =u_{1} \\
\dot{y} & =\tan \theta u_{1} \\
\dot{\phi} & =u_{2} \\
\dot{\theta} & =\frac{1}{l} \sec \theta \tan \phi u_{1}
\end{aligned}
$$



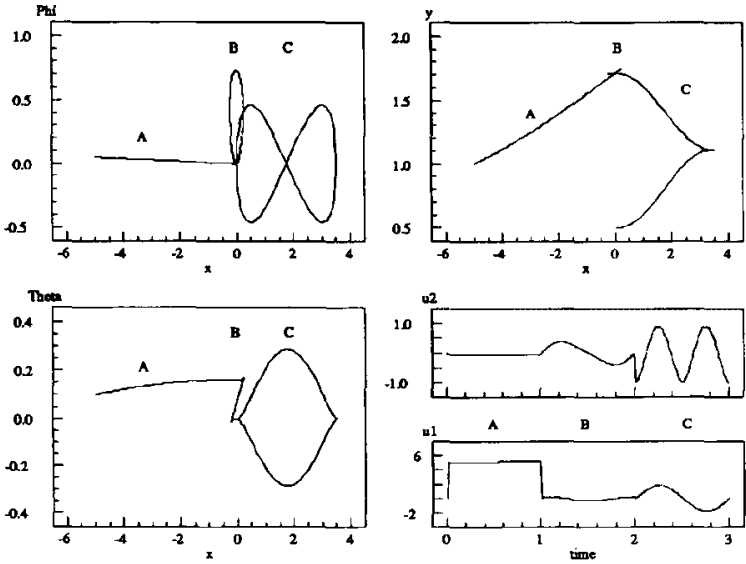

Figure 1: Sample trajectories for steering a $(2,1,1)$ system. The trajectory shown is a three stage path which moves the system from the initial configuration to the origin.

Choose the $y$ position of the car as the function $h$; it is easy to verify that this function satisfies the conditions of Proposition 4. The resulting change of coordinates is

$$
\begin{array}{ll}
\xi_{1}=x & u_{1}=v_{1} \\
\xi_{2}=\frac{1}{l} \sec ^{3} \theta \tan \phi & u_{2}=-\frac{3}{l} \sin ^{2} \phi \sin \theta v_{1}+\frac{1}{l} \cos ^{2} \theta \cos ^{3} \phi v_{2} \\
\xi_{3}=\tan \theta & \\
\xi_{4}=y &
\end{array}
$$

And the transformed system has the form:

$$
\begin{aligned}
& \dot{\xi}_{1}=v_{1} \\
& \dot{\xi}_{2}=v_{2} \\
& \dot{\xi}_{3}=\xi_{2} v_{1} \\
& \dot{\xi}_{4}=\xi_{3} v_{1}
\end{aligned}
$$

This system can now be steered using the sinusoidal algorithm of the previous section or another method, such as Lafferriere and Sussmann's algorithm for generating motions for nilpotent systems. The motion is implemented as a feedback pre-compensator which converts the $v$ inputs into the actual system inputs, $u$. This feedback transformation agrees with that used in Lafferriere and Sussmann to nilpotentize the kinematic car example. Their formulation of the feedback transformation was not presented, although it seems clear that a similar approach must have been used.

Figure 1 shows the results of using chained form to steer an automobile. These trajectories are qualitatively similar to those in [21], but do not require the calculation of Fourier coefficients for determining open loop trajectories.

Example 2 (Car with $N$ trailers) Consider first the case of a car pulling a single trailer. The equations of motion are identical to those of the car, with an additional equation specifying the motion of the attached trailer [22]:

$$
\dot{\theta}_{1}=\sin \left(\theta_{0}-\theta_{1}\right) u_{1}
$$

By solving the partial differential equations in the statement of the proposition above, it can be shown that the function

$$
h\left(y, \theta_{1}\right)=y-\log \left(\frac{1+\sin \theta_{1}}{\cos \theta_{1}}\right)
$$

generates a chained set of coordinates. Again we can locally steer the trailer using sinusoidal inputs or other methods.

When additional trailers are added, the distribution $\Delta_{1}$ is no longer involutive and hence the procedure outlined above does not apply. Since the conditions in the proposition are only sufficient conditions, this does not mean that a car with $N$ trailers cannot be steered using sinusoids. But a more complicated change of basis would be required in order to convert the vector fields to the necessary form. This example points out the weaknesses of the theorem and provides directions for future research.

\section{Discussion and extensions}

The conditions given in Proposition 2 give a constructive set of sufficient conditions for converting a nonholonomic control system into chained form. Using chained form, it is possible to efficiently find paths for steering a system between arbitrary configurations. We have presented one such method based on sinusoidal inputs although other techniques can be used. We have presented only the simplest case ( 2 inputs, 1 non-zero length chain) here, but it is possible to extend this result in several directions. The method proposed here is also useful in the more general area of local nilpotentization of distributions of vector fields [7]. In particular, if the conditions of Proposition 2 hold for a set of two vector files $g_{1}$ and $g_{2}$ on $\mathbb{R}^{n}$, then there is a nilpotent basis for the corresponding distribution.

Converting a system to chained form is very closely related to the exact linearizability conditions for a general nonlinear system. As we noted in the introduction, linear control techniques cannot be applied to nonholonomic control systems due to the lack of a drift term. However, many of the underlying geometric tools on which exact linearization techniques are based can be applied effectively to nonholonomic systems. We see this in the application of Proposition 2. In particular, we note that if the distribution

$$
\left\{g_{2}, \operatorname{ad}_{g_{1}} g_{2}, \cdots, \operatorname{ad}_{g_{1}}^{n_{x}-1} g_{2}\right\}
$$

is involutive, then we are guaranteed of the existence of a function $h$ which annihilates the distribution. Finding a specific $h$ which satisfies this requires solving a set of first order partial differential equations

There are many open questions which are currently being studied by ourselves and others. These include the introduction of a drift vector field into the control system and feedback control of nonholonomic systems. Some initial results in these areas can be found in the work of Bloch and McClamroch [2, 3] and Samson [2i]. We also note that approximate versions of Proposition 4 can be formulated using tools similar to those developed by Krener $[10,11]$.

\section{Acknowledgements}

The authors would like to thank Richard Montgomery, Jean-Paul Laumond, Hector Sussmann and the members of the UC Berkeley Robotics Lab for many useful discussions in the area of nonholonomic motion planning.

\section{References}

[1] J. Barraquand and J-C. Latombe. On nonholonomic mobile robots and optimal maneuvering. In 4th International Symposium on Intelligent Control, Albany, NY, 1989. 
[2] A. M Bloch and N. H. McClamroch. Control of mechanical systems with classical nonholonomic constraints. In IEEE Control and Decision Conference, pages 201-205, 1989.

[3] A. M Bloch and N. H. McClamroch. Controllability and stabilizability properties of a nonholonomic control system. In IEEE Control and Decision Conference, 1990.

[4] R. W. Brockett. Control theory and singular Riemannian geometry. In New Directions in Applied Mathematics, pages 11-27. Springer-Verlag, New York, 1981.

[5] V. Gershkovich and A. Vershik. Nonholonomic manifolds and nilpotent analysis. Journal of Geometry and Physics, $5(3): 407-452,1988$.

[6] M. Grayson and R. Grossman. Models for free nilpotent Lie algebras. Technical Memo PAM-397, Center for Pure and Applied Mathematics, University of California, Berkeley, 1987. (to appear in J. Algebra).

[7] H. Hermes, A. Lundell, and D. Sullivan. Nilpotent bases for distributions and control systems. Journal of Differential Equations, 55:385-400, 1984.

[8] A. Isidori. Nonlinear Control Systems. Springer-Verlag, 2nd edition, 1989.

[9] P. Jacobs, J-P. Laumond, M. Taix, and R. Murray. Fast and exact trajectory planning for mobile robots and other systems with non-holonomic constraints. Technical Report 90318, LAAS/CNRS, Toulouse, France, September 1990.

[10] A. J. Krener. Approximate linearization by state feedback and coordinate change. Systems and Control Letters, 5:181$185,1984$.

[11] A. J. Krener, S. Karahan, M. Hubbard, and R. Frezza. Higher order linear approximations to nonlinear control systems. In IEEE Control and Decision Conference, pages 519-523, 1987.

[12] G. Lafferriere and H. J. Sussmann. Motion planning for controllable systems without drift. In IEEE International Conference on Robotics and Automation, pages 1148-1153, 1991.

[13] J-P. Laumond. Feasible trajectories for mobile robots with kinematic and environment constraints. In Intelligent $A u$ tonomous Systems. North Holland, 1987.

[14] J-P. Laumond. Finding collision-free smooth trajectories for a non-holonomic mobile robot. In International Joint Conference on Artificial Intelligence, pages 1120-1123, 1987.

[15] J-P. Laumond and T. Siméon. Motion planning for a two degrees of freedom mobile robot with towing. In IEEE International Conference on Control and Applications, 1989.

[16] $\mathrm{Z} . \mathrm{Li}$ and $\mathrm{J}$. Canny. Motion of two rigid bodies with rolling constraint. IEEE Transactions on Robotics and Automation, $6(1): 62-71,1990$.

[17] Z. Li, R. Montgonery, and M. Raibert. Dynamics and optimal control of a legged robot in flight phase. In IEEE International Conference on Robotics and Automation, pages 1816-1821, 1989

[18] R. Montgomery. Isoholonomic problems and some applications. Communications in Mathematical Physics, 128:565$592,1990$.

[19] R. M. Murray. Robotic Control and Nonholonomic Motion Planning. PhD thesis, University of California at Berkeley, 1990.

[20] R. M. Murray and S. S. Sastry. Grasping and manipulation using multifingered robot hands. In R. W. Brockett, editor, Robotics: Proceedings of Symposia in Applied Mathematics, Volume 41, pages 91-128. American Mathematical Society, 1990.

[21] R. M. Murray and S. S. Sastry. Steering nonholonomic systems using sinusoids. In IEEE Control and Decision Conference, 1990.
[22] R. M. Murray and S. S. Sastry. Nonholonomic motion planning: Steering using sinusoids. Technical Report UCB/ERL M9l/45, Electronics Research Laboratory, University of California at Berkeley, 1991 .

[23] D. Nguyen and $\mathbf{B}$. Widrow. Neural networks for self-learning control systems. IEEE Control Systems Magazine, 10(3):18$23,1990$.

[24] C. Samson. Velocity and torque feedback control of a nonholonomic cart. In International Workshop in Adaptive and Nonlinear Control: Issues in Robotics, 1990.

[25] N. Sreenath, Y. G. Oh, P. S. Krishnaprasad, and J. E. Marsden. The dynamics of coupled planar rigid bodies. part i: Reduction, equilibria and stability. Dynamics and Stability of Systems, 3(1 \& 2), 1988.

[26] H. J. Sussmann and W. Liu. Limits of highly oscillatory controls and the approximation of general paths by admissible trajectories. Technical Report SYCON-91-02, Rutgers Center for Systems and Control, 1991. 